\title{
Reparación Ósea en Alvéolos de Ratas Ovariectomizadas con Biosilicato Cristalizado. Análisis Histomorfométrico
}

\author{
Alveolar Bone Repair in Ovarictomized Rats with \\ Crystallized Biosilicate. Histomorphometric Analysis
}

Marcelo Rodrigues Azenha'; Osvaldo Magro Filho²; Oscar Peitt ${ }^{3}$; Balter Toro ${ }^{4}$ \& Alberto Rodríguez Archilla ${ }^{1}$

\begin{abstract}
AZENHA, M. R.; MAGRO FILHO, O.; PEITL, O.; TORO, B. \& ARCHILLA A. R. Reparación ósea en alvéolos de ratas ovariectomizadas con biosilicato cristalizado. Análisis histomorfométrico. Int. J. Odontostomat., 13(2):162-171, 2019.

RESUMEN: Evaluar el proceso de reparación alveolar en ratas sometidas a cirugía de simulacro u ovariectomizadas tras el relleno alveolar con coágulo o con biosilicato cristalino. Sesenta ratas Wistar fueron divididas en cuatro grupos $(n=15)$ de acuerdo con el tratamiento: Grupo 1- ratas sometidas a cirugía de simulacro con alveolos rellenados con coágulo; Grupo 2- ratas sometidas a cirugía de simulacro con alveolos rellenados con biosilicato cristalino; Grupo 3- ratas ovariectomizadas con alveolos rellenados con coágulo; Grupo 4- ratas ovariectomizadas con alveolos rellenados con biosilicato cristalino. Después de 7, 14 y 28 días, los animales fueron sacrificados, se tomaron muestras óseas que fueron teñidas con hematoxilina-eosina y analizadas al microscopio para realizar un análisis histomorfométricos. Los mayores porcentajes de formación de hueso se presentaron en los grupos 1 (32 \% a los 7 días, $46 \%$ a los 14 días y $83.5 \%$ a los 28 días) y 4 (27,1 $\%$ a los 7 días, $41,1 \%$ a los 14 días y $79,7 \%$ a los 28 días). En los alveolos rellenados con coágulo, las ratas sometidas a cirugía de simulacro mostraron los mejores resultados, mientras que, en los alveolos rellenados con biosilicato, las ratas ovariectomizadas tenían porcentajes significativamente mayores. En este estudio, el biosilicato cristalino se comportó como un biomaterial adecuado para la reparación ósea, favoreciendo la osteoconducción.
\end{abstract}

PALABRAS CLAVE: histomorfometría, osteoporosis, ratas Wistar, silicatos.

\section{INTRODUCCIÓN}

Los substitutos óseos han sido ampliamente utilizados con el objetivo de minimizar las limitaciones que aparecen después de grandes pérdidas de estructuras óseas o en los casos de tejidos óseos de baja calidad (Wang \& Yeung, 2017).

Según Hasan et al. (2018), estos biomateriales deben ser oseointegrables, es decir, tener la capacidad de unirse químicamente a la superficie del lecho óseo receptor con ausencia de tejido fibroso;osteoconductores, sosteniendo el crecimiento óseo en la superficie; osteoinductores, promoviendo la diferenciación de células mesenquimales pluripotenciales del lecho receptor en células osteoblásticas y deben todavía presentar la capacidad de ser osteogénicos para que ocurra la neoformación ósea por las células osteoblásticas presentes en el biomaterial.

El injerto de hueso autógeno es el único que satisface todos estos requisitos, mientras que, los substitutos óseos artificiales o sintéticos solamente presentan las propiedades de oseointegración y osteoconducción (Costantino \& Friedman, 1994; Cypher \& Grossman, 1996).

Idealmente los materiales artificiales deben ser biocompatibles, no tóxicos, activos y deben sufrir remodelación, favoreciendo la neoformación de tejido óseo. Mecánicamente tales biomateriales deben tener resistencia y elasticidad similares al lecho receptor o tejido óseo, debiendo ser de fácil aplicación,

\footnotetext{
${ }^{1}$ Universidad de Granada, Granada, España.

2 Facultad de Odontología, UNESP, Araçatuba, Brasil.

${ }^{3}$ Universidad Federal de São Carlos, Brasil.

${ }^{4}$ Reabilitación Oral, USP, Ribeirão Preto, Brasil.
} 
adaptación y estar disponibles en cantidad adecuada y en cualquier tiempo. Por este motivo, se han desarrollado numerosas investigaciones buscando la obtención de biomateriales que presenten características semejantes al hueso humano y que puedan ser utilizados en diferentes situaciones (Pina et al., 2018).

Recientemente se ha desarrollado en la Universidad Federal de San Carlos (UFSCar) en Brasil, un biovidrio perteneciente al sistema $\mathrm{Na}_{2} \mathrm{O}-\mathrm{CaO}-\mathrm{SiO}_{2}-$ $\mathrm{P}_{2} \mathrm{O}_{5}, 100 \%$ cristalino denominado "Biosilicato ${ }^{\circledR}$ " (PI 0300644-1) para la aplicación en áreas médicas y odontológicas. Este biovidrio viene siendo evaluado como sustituto óseo en diferentes situaciones, presentando resultados prometedores para ser usados en la clínica odontológica (Moura et al., 2007; Azenha et al., 2010; Roriz et al., 2010; Azenha et al., 2015). Los resultados sugieren que el biosilicato cristalino es biocompatible cuando se coloca en el interior de alvéolos dentales y que esta biocompatibilidad puede aumentar la formación ósea después de la extracción dental.

Teófilo et al. (2003) estudiaron el comportamiento del biovidrio en alvéolos de ratas con osteoporosis experimental inducida mediante ovariectomía, concluyendo que las mismas presentaron un retardo acentuado en la formación de nuevo hueso. Los estudios de la formación ósea en pequeños animales con condiciones sistémicas alteradas, como la osteoporosis son de extrema importancia para el desarrollo de nuevos biomateriales (Wu et al., 2016).

El objetivo de este trabajo fue evaluar el proceso de reparación alveolar de ratas ovariectomizadas después del llenado con biosilicato cristalino a través de un análisis clínico e histomorfométrico.

\section{MATERIAL Y MÉTODO}

Animales de experimentación. Para este estudio se utilizaron sesenta ratas hembra (Rattus norvegicus, variedad Wistar), con pesos entre 200 y 300 gramos procedentes del Bioterio de la Facultad de Odontología de Ribeirão Preto (Brasil). Según la técnica quirúrgica empleada, se establecieron dos intervenciones:

Treinta ratas fueron sometidas a ovariectomía y otras treinta fueron incluidas en el grupo de cirugía de simulacro (sham surgery).
Ovariectomía. El grupo de ratas sometidas a ovariectomía fueron intervenidas por vía dorsal según el siguiente protocolo:

1. Se anestesia del animal con isoflurano al $1,5 \%$ con una mezcla de oxígeno/óxido nitroso $30 \%$ : $70 \%$ y se coloca en posición prona sobre una placa calefactora conectada a un termómetro rectal manteniendo la temperatura corporal dentro de los $37 \pm 0,5^{\circ} \mathrm{C}$.

2. Se administra carprofeno $5 \mathrm{mg} / \mathrm{kg}$ de peso corporal diluido en solución salina por vía subcutánea en la cara lateral del cuello del animal para el alivio del dolor postoperatorio.

3. Se afeita un área de $4 \times 4 \mathrm{~cm}$ cefálicamente desde la cresta ilíaca. Posteriormente se lava el área afeitada con solución antiséptica.

4. Se realiza una incisión de $2-3 \mathrm{~cm}$ en la línea media con disección de la piel de la fascia subyacente. Se hace una nueva incisión a través de la fascia, un centímetro lateral de la línea media. A continuación se realiza una disección roma lateralmente debajo de esta fascia hasta llegar a la cavidad abdominal.

5. Con unas pinzas se agarra el tejido adiposo que rodea al ovario en la cavidad abdominal y se saca. Se identifica el ovario y se ligan los cuernos uterinos y los vasos a $0,5-1 \mathrm{~cm}$ proximalmente de esta estructura. Seguidamente se corta el ovario y se liga el tejido adiposo, aposicionando el tejido restante de vuelta a la cavidad abdominal.

6. Se cierran las heridas con una sutura monofilamento. Tras la recuperación de la anestesia, se coloca al animal en una jaula calentada a unos 25-27 ${ }^{\circ} \mathrm{C}$ durante al menos 2 horas después de la cirugía.

7. Veinticuatro horas después de la cirugía, se inyecta al animal por vía subcutánea carprofeno $5 \mathrm{mg}$ $/ \mathrm{kg}$ de peso corporal diluidos en solución salina.

En las ratas sometidas a la ovariectomía, después de la cirugía, su dieta fue sustituida por una ración especial con calcio al 0,1\% y fósforo al 0,5\% con el objetivo de disminuir la masa ósea de estos animales. Después de 6 semanas tras la ovariectomía y las cirugías de simulacro, en cada animal se procedió a la extracción de incisivo central superior. A continuación, las ratas fueron distribuidas de acuerdo con los siguientes cuatro grupos: 
Grupo 1. (Cirugía de simulacro + Coágulo). Compuesto por 15 ratas sometidas a cirugía de simulacro con el alvéolo relleno de coágulo.

Grupo 2. (Cirugía de simulacro + Biosilicato cristalino). Constituido por 15 ratas sometidas a la cirugía de simulacro con el alvéolo rellenado por biosilicato cristalino.

Grupo 3. (Ovariectomía + Coágulo). Formado por 15 ratas sometidas a la cirugía de simulacro con el alvéolo relleno de coágulo.

\section{Grupo 4. (Ovariectomía + Biosilicato cristalino).}

Configurado por 15 ratas sometidas a la cirugía de ovariectomía con el alvéolo rellenado por biosilicato cristalino.

Todos los procedimientos se realizaron de acuerdo con las reglas del Consejo Brasileño de Control de Experimentación Animal (CONCEA) y fueron aprobados por el Comité Ético de Uso y Bienestar Animal (CEUA) de la Universidad de Sao Paulo de Brasil (número 11.1.211.53.5).

Biomaterial utilizado. El biosilicato cristalino (Biosilicate $®$ ) es una vitro-cerámica bioactiva totalmente cristalizada del sistema cuaternario con la siguiente fórmula $\mathrm{Na}_{2} \mathrm{O}-\mathrm{CaO}-\mathrm{SiO}_{2}-$ $\mathrm{P}_{2} \mathrm{O}_{5}$ proporcionada por el Laboratorio de Materiales Vítreos (LaMaV) del Departamento de Ingeniería de Materiales, Universidad Federal de San Carlos, Sao Paulo, Brasil. Está compuesto por sílice de alta pureza junto con carbonato de calcio, carbonato de sodio y fosfato de sodio. Es un biomaterial relativamente fácil de cortar y perforar que permite la fabricación de implantes con diferentes formas para propósitos específicos.

Estudio histomorfométrico. Para cada grupo, se establecieron 3 períodos de evaluación a los 7, 14 y 28 días de la extracción dental en los animales. En cada grupo $(n=15)$, se sacrificaron 5 ratas en cada uno de los 3 períodos evaluados: a los 7 días $(n=5)$, a los 14 días $(n=5)$ y a los 28 días $(n=5)$. Se procedió al procesamiento histológico para lo que se obtuvieron 20 cortes distanciados uno del otro por un intervalo de $60 \mathrm{~mm}$ de cada región a ser estudiada. Cada alvéolo fue dividido en tres tercios: coronal, medio y apical. Las muestras fueron teñidas con la tinción clásica de hematoxilina-eosina. El análisis cuantitativo de cada muestra tisular se realizó con un microscopio Leica DM LB2 (Leica Microsystems Wetzlar $\mathrm{GmbH}$, Wetzlar, Germany) con una cámara digital Leica DFC 280 (Leica Microsystems Wetzlar GmbH, Wetzlar, Germany), siendo las imágenes procesadas con el programa "DFCTwain for PC versión 7.7.1" (Leica Microsystems Wetzlar GmbH, Wetzlar, Germany), obteniéndose la fracción de volumen del hueso neoformado y el porcentaje de tejido conectivo en el interior del alvéolo. Los cortes histológicos fueron visionados al microscopio con objetivos de 20X y 40X y, en cada alvéolo, se analizaron 15 campos microscópicos (5 campos en cada tercio) con la ayuda de una rejilla tipo Merz colocada sobre los cortes. Esta cuadrícula modificada consiste en un cuadrado que limita el área de prueba y que contiene 100 cruces de líneas que se utilizan para contar puntos sobre una determinada estructura histológica. Conociendo el área cubierta por la rejilla es posible estimar la densidad volumétrica del tejido óseo en los diferentes períodos de estudio.

Estudio Estadístico. El análisis estadístico se realizó con el programa estadístico Assistat para Windows, versión portuguesa (Silva FAS, Universidad Federal de Campina Grande, Brasil) con prueba no paramétrica de Mann-Whitney $(p<0,05)$.

\section{RESULTADOS}

Estudio Clínico. En el estudio clínico no se observó ninguna señal clínica de inflamación o infección, permaneciendo los animales clínicamente sanos.

\section{Estudio Histomorfométrico}

\section{Evaluación a los 7 días}

Grupo 1: Cirugía de simulacro + Coágulo- Tejido conjuntivo cicatricial rico en fibroblastos, capilares y hueso neoformado. El alvéolo aparecía parcialmente rellenado por delicadas trabéculas óseas inmaduras y coágulo sanguíneo en organización (Fig. 1).

Grupo 2: Cirugía de simulacro + BiosilicatoTrabéculas óseas inmaduras en proceso de formación contigua al material utilizado, conteniendo todavía tejido conjuntivo con bajas cantidades de coágulos sanguíneos (Fig. 2).

Grupo 3: Ovariectomía + Coágulo- Gran cantidad de tejido conjuntivo, coágulos, trabéculas óseas inmaduras y muchos fibroblastos (Fig. 3).

Grupo 4: Ovariectomía + Biosilicato- Extensas áreas de tejido conjuntivo y coágulos en las proximidades del biosilicato con formación de nuevas trabéculas óseas (Figs. 4a y b). 


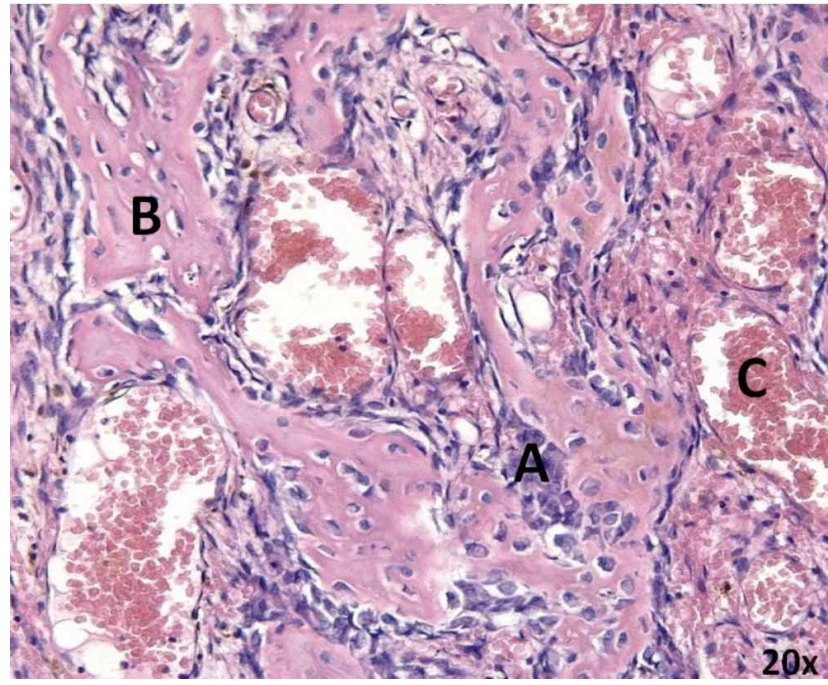

Fig. 1. Grupo de cirugía de simulacro + Coágulo a los 7 días. Tejido conectivo rico en fibroblastos, capilares y huesonuevo (trabéculas óseas). A: Tejido conectivo; $\mathrm{B}$ : Hueso recién formado; C: Coágulo. (H-E 20X).

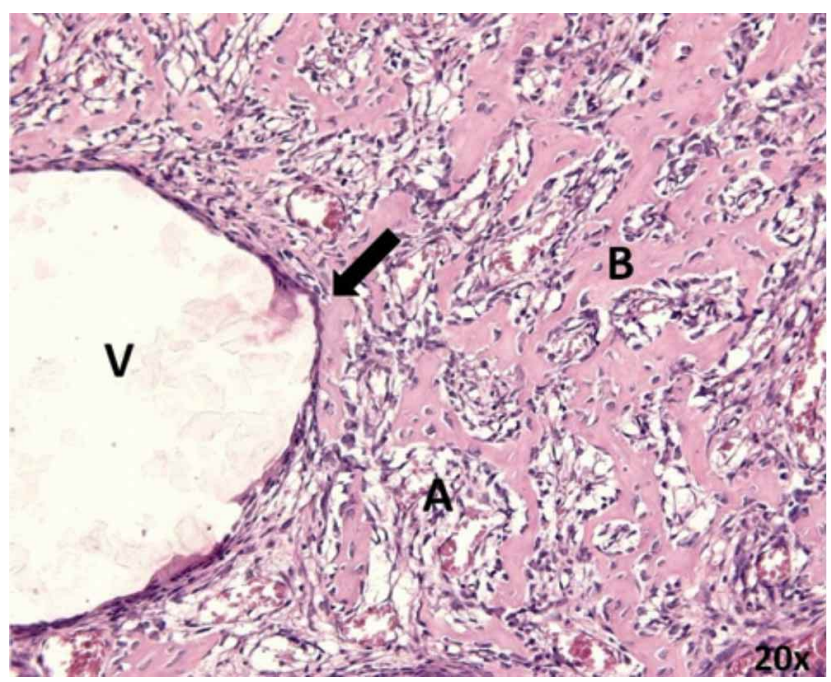

Fig. 2. Grupo de cirugía de simulacro + Biosilicato a los 7 días. Trabéculas óseas inmaduras en contacto con el Biosilicato (flecha) y tejido conectivo con poca cantidad de coágulo sanguíneo. A: Tejido conectivo; $\mathrm{B}$ : Hueso recién formado; V: Partícula del Biosilicato. (Tinción H-E 20X).

\section{Evaluación a los 14 días}

Grupo 1: Cirugía de simulacro + CoáguloTrabéculas óseas más gruesas envueltas por osteoblastos (Fig. 5).

Grupo 2: Cirugía de simulacro + Biosilicato- Disminución de la cantidad de tejido conjuntivo y un estrecho contacto de la superficie del biosilicato con el tejido óseo neoformado (Fig. 6).

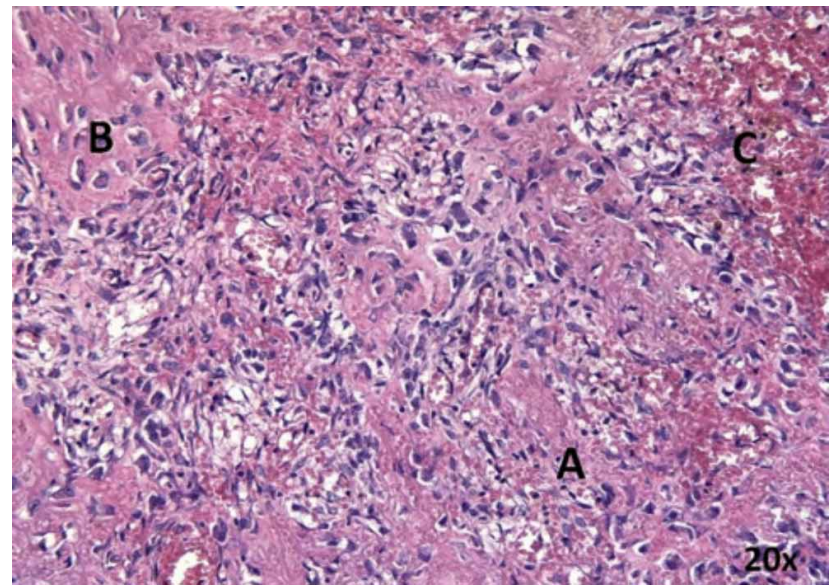

Fig. 3. Grupo ovariectomía + Coágulo a los 7 días. Tejido conectivo y coágulo en contacto con las partículas del biomaterial. Início de la formación ósea. A: Tejido conectivo; B: Hueso; C: Coágulo. (H-E 20X).

Grupo 3: Ovariectomía + Coágulo- Disminución del tejido conectivo y pequeñas áreas de coágulo. La mayor parte del defecto aparecía rellenado por hueso (Fig. 7).

Grupo 4: Ovariectomía + Biosilicato- Tejido óseo en íntimo contacto con el biosilicato, siendo considerado biocompatible y sin efectos negativos (Fig. 8).

\section{Evaluación a los 28 días}

Grupo 1: Cirugía de simulacro + Coágulo-Se encontró una completa formación ósea, con pequeñas cantidades de tejido conjuntivo maduro (Fig. 9).

Grupo 2: Cirugía de simulacro + Biosilicato-Hueso tanto en contacto con el biosilicato como entre las partículas del mismo. El biosilicato cristalino presentó biocompatibilidad y osteointegración (Fig. 10).

Grupo 3: Ovariectomía + Coágulo- Acentuada formación de hueso, con pequeñas áreas de tejido conectivo en el interior del tejido mineralizado y coágulos (Fig. 11).

Grupo 4: Ovariectomía + Biosilicato-El tejido óseo neoformado se encontró en toda la extensión del alvéolo y en contacto con el biomaterial (Fig. 12).

Análisis Histométrico. En la Tabla I se muestra el tejido óseo (expresado en porcentajes) encontrado en los 3 períodos evaluados tras la extracción dental ( 7 , 14 y 28 días). 


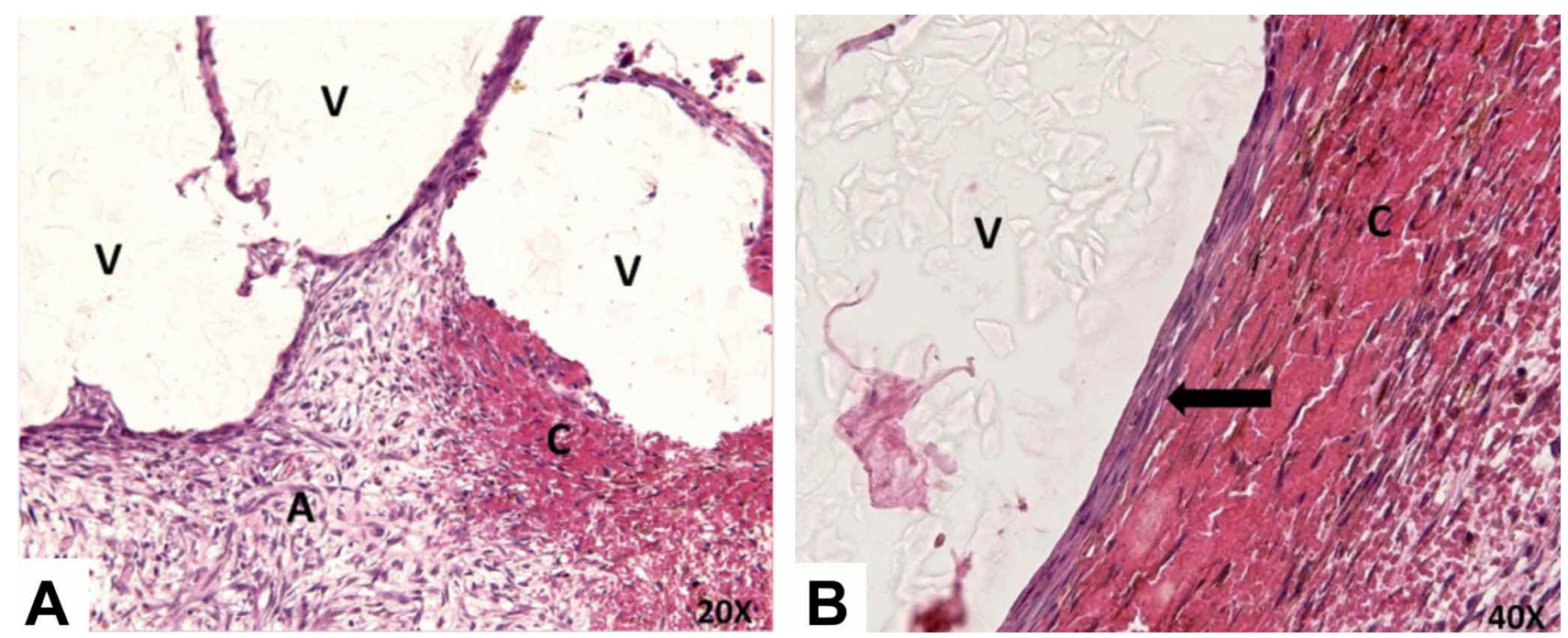

Fig. 4. A. Grupo ovariectomía + Biosilicato a los 7 días. Presencia de áreas con coágulo en la proximidad de los gránulos de Biosilicato. A: Tejido conectivo; C: Coágulo; V: Partículas del Biosilicato. (H-E 20X). B. Grupo ovariectomía + Biosilicato a los 7 días. Extensa área de tejido conectivo (flecha) y la presencia de áreas con en la proximidad de los gránulos de Biosilicato. A: Tejido conectivo; C: Coágulo; V: Partículas del Biosilicato. (H-E 40X).

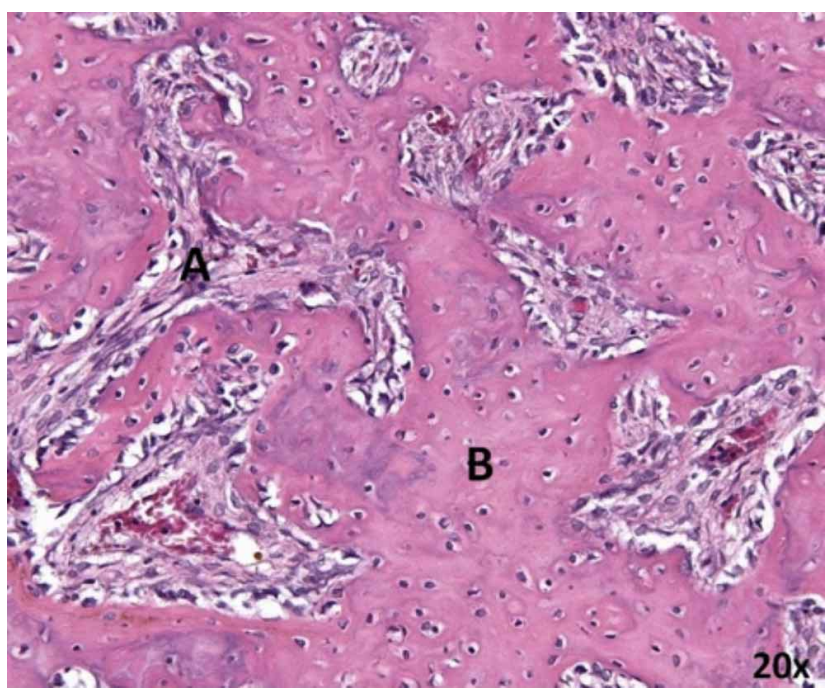

Fig. 5. Grupo de cirugía de simulacro + Coágulo a los 14 días. Trabéculas de hueso maduro y tejido conectivo y coágulo en menor cantidad. A: Tejido conectivo; B: Hueso. (H-E 20X).

A los 7 días de evaluación, los grupos 1 y 4 presentaron los mejores resultados (32\% y $27,1 \%$ respectivamente, aunque sin diferencias estadísticamente significativas $(p>0,05)$ entre ellos. Por el contrario, entre los grupos $2(19,4 \%)$ y 3 $(13,7 \%)$ sí se encontró relación estadísticamente significativa $(p<0,05)$. A los 14 días de evaluación, no se observó asociación estadísticamente signifi-

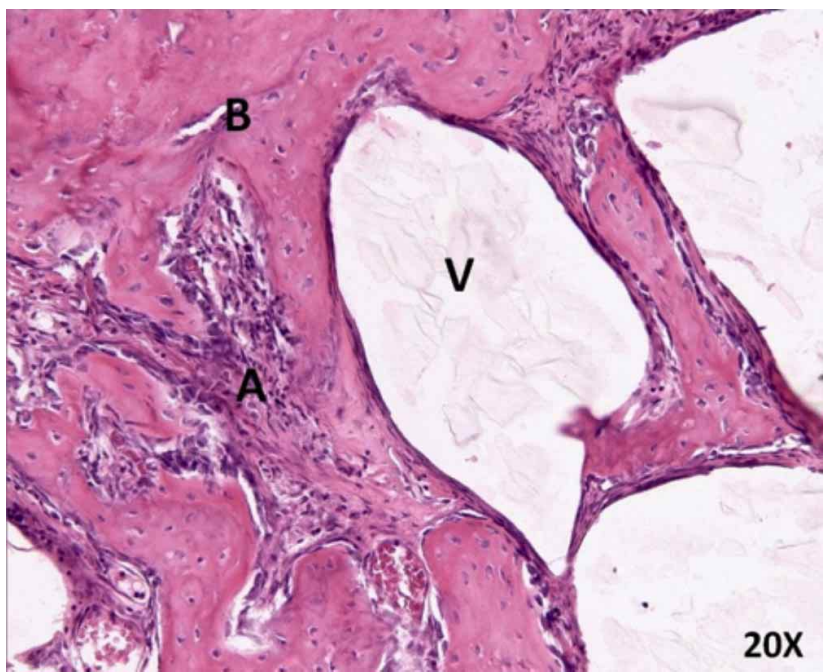

Fig. 6. Grupo de cirugía de simulacro + Biosilicato a los 14 días. Menor cantidad de tejido conectivo e íntimo contacto entre el biosilicato y el tejido óseo recién formado. A: Tejido conectivo; B: Hueso; V: Biosilicato. (H-E 20X).

cativa entre los grupos 1 (46\%) y $4(41,1 \%)$. Sin embargo, se hallaron diferencias estadísticamente significativas $(p<0,05)$ al comparar los grupos $2(32,5$ $\%)$ y $3(26 \%)$. Finalmente, a los 28 días de evaluación, se constató relación estadísticamente significativa $(p<0,05)$ entre los grupos $1(83,5 \%)$ y $3(60,5$ $\%)$ y entre los grupos $2(64,2 \%)$ y $4(79,7 \%)$ entre sí. 


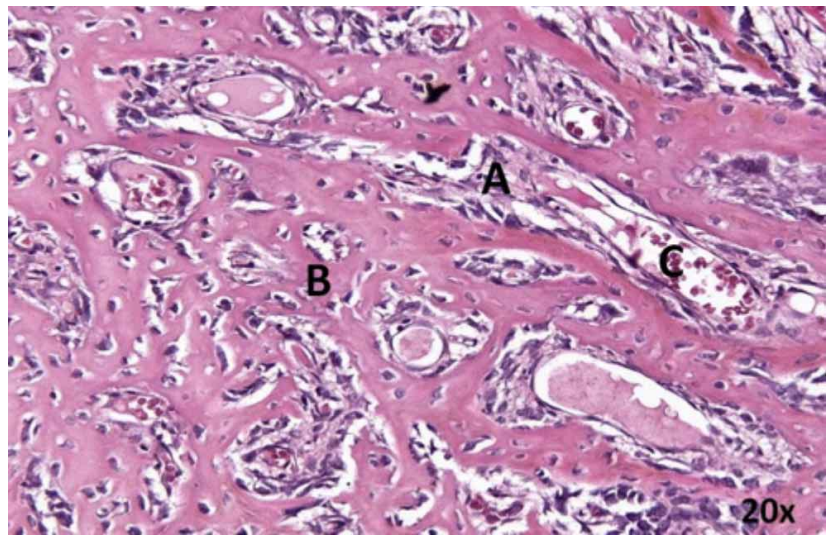

Fig. 7. Grupo ovariectomía + Coágulo a los 14 días.Trabéculas de tejido óseo entremezcladas por tejido conectivo y pequeñas áreas de coágulo. A: Tejido conectivo; B: Hueso; C: Coágulo. (H-E 20X)

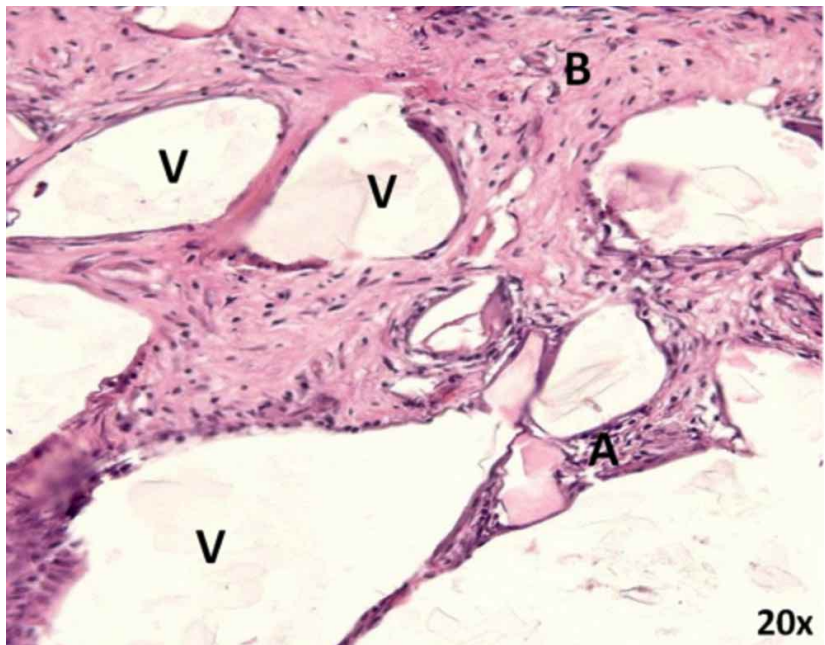

Fig. 8. Grupo ovariectomía + Biosilicato a los 14 días. Partículas de biosilicato en contacto directo con el tejido óseo recién formado. Pocas áreas de tejido conectivo. A: Tejido conectivo; B: Hueso recién formado; V: Partículas del Biosilicato. (H-E 20x).

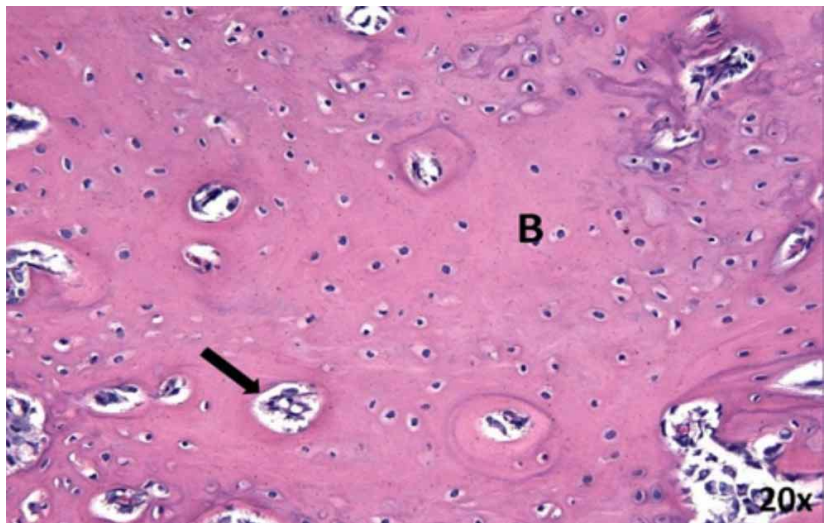

Fig. 9. Grupo de cirugía de simulacro + Coágulo a los 28 días. Formación ósea completa con poca cantidad de tejido conectivo maduro (flecha). B: Hueso. (H-E 20x).

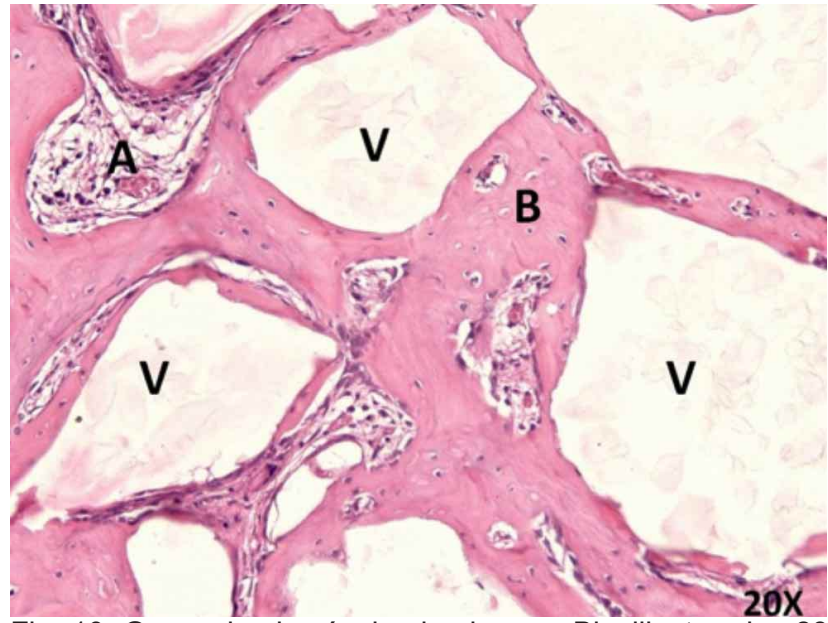

Fig. 10. Grupo de cirugía de simulacro + Biosilicato a los 28 días. Tejido óseo en íntimo contacto con la superfície y entre las partículas de biosilicato. Tejido conectivo en poca cantidad. A: Tejido conectivo; B: Hueso; V: Partículas de biosilicato.(H-E 20x).

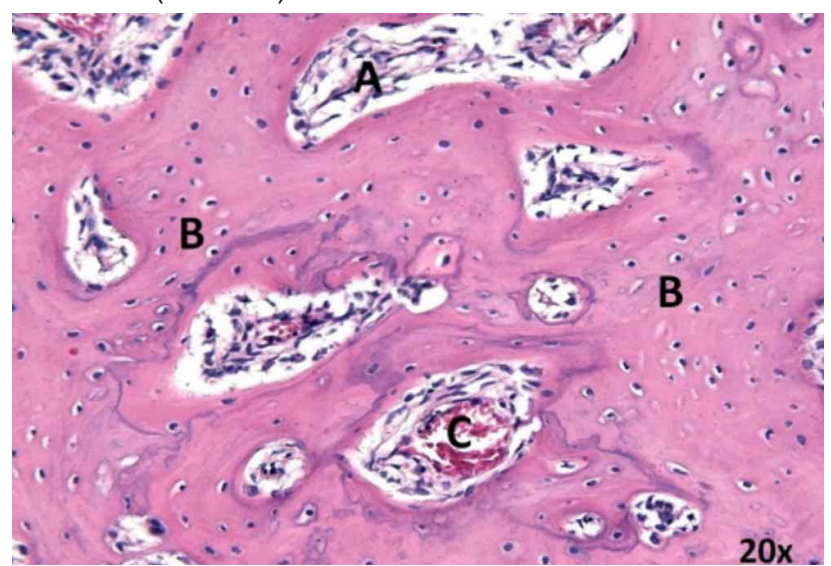

Fig. 11. Grupo ovariectomía + Coágulo a los 28 días.Extensa formación de hueso con discretas áreas de tejido conectivo y coágulo. A: Tejido conectivo; B: Hueso; C: Coágulo.(H-E 20x).

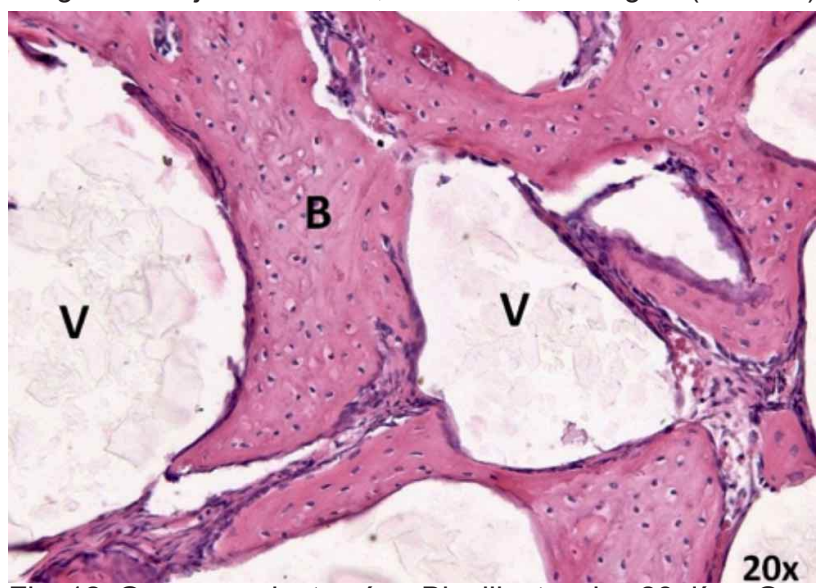

Fig. 12. Grupo ovariectomía + Biosilicato a los 28 días. Gran cantidad de tejido óseo recién formado en toda la extensión del alvéolo y en contacto con fragmentos de biosilicato. B: Hueso maduro; V: Partículas de biosilicato. (H-E 20x). 
Tabla I. Porcentaje (\%) de tejido óseo después de 7, 14 y 28 días tras la colocación del biomaterial o del coágulo.

\begin{tabular}{llll}
\hline & \multicolumn{3}{l}{ Perío dos de evaluación } \\
\cline { 2 - 4 } Grupos & 7 días & 14 días & 28 días \\
\hline 1. CS + Coágulo & $32 \%$ & $46 \%$ & $83,5 \%{ }^{\mathrm{a}}$ \\
2. CS + Biosilicato & $19,4 \%^{\mathrm{a}}$ & $32,5 \%^{\mathrm{a}}$ & $64,2 \%{ }^{\mathrm{b}}$ \\
3. OV + Coágulo & $13,7 \%^{\mathrm{a}}$ & $26 \%^{\mathrm{a}}$ & $60,5 \%^{\mathrm{a}}$ \\
\hline
\end{tabular}

\section{DISCUSIÓN}

Los defectos resultantes de traumas, ocasionados por resecciones óseas como consecuencia de patologías y en la recuperación de márgenes o áreas reabsorbidas con el paso del tiempo que necesiten de rehabilitación, son algunos de los ejemplos donde los substitutos óseos pueden ser aplicados.

Frente a esas necesidades y con el fin de disminuir la morbilidad que las técnicas autógenas pueden causar en los individuos, el objetivo de este estudio fue evaluar la respuesta biológica de un biosilicato cristalino recientemente desarrollado y que ha presentado resultados favorables. El Bioglass $45 S 5 \AA$ es considerado el vidrio bioactivo que presenta las mejores características para uso en el área de salud, siendo ampliamente probado en situaciones in vitro e in vivo como control de otros biomateriales.

Cuando las partículas de los biovidrios presentan en su superficie la formación de capas de hidroxiapatita, permiten que los osteoblastos y los constituyentes orgánicos sean atraídos, con la consecuente formación de un sustrato con composición química semejante al tejido óseo (Azenha et al., 2010). La completa cristalización del biovidrio (biosilicato cristalino) y la aplicación de este nuevo biosilicato cristalino en las investigaciones ha demostrado que este material sintético presenta características similares o incluso superiores al Bioglass45S5® (Granito et al., 2009). Este biomaterial fue inicialmente utilizado y probado en el tratamiento de la hipersensibilidad dentinaria. Además también ejerce actividad antimicrobiana, lo que puede ser considerado como una gran ventaja cuando se utiliza en las grandes reconstrucciones maxilares por la amplia gama de microorganismos presentes en la cavidad bucal (Tirapelli et al., 2011; Martins et al., 2011).

Sin embargo, pocos estudios han evaluado el comportamiento de los biovidrios en ratas ovariectomizadas y con dietas pobres en calcio. El desarrollo del presente estudio se basó en los trabajos desarrollados por Teófilo et al. siendo los procedimientos quirúrgicos y el tratamiento dispensado a los animales ejecutado de forma bastante similar. En ese trabajo los autores afirman que la cirugía de ovariectomía asociada a la dieta pobre en calcio induce la pérdida ósea con el consiguiente desarrollo de la osteoporosis dos veces más pronunciada en el hueso alveolar que en la región de la tibia. Según sus conclusiones, la masa ósea sufre una pérdida de hasta $35 \%$ en la quinta semana después de la ovariectomía y tiende a continuar hasta la $11^{\mathrm{a}}$ semana. En nuestro estudio, la extracción de los incisivos superiores y la implantación del biosilicato cristalino fueron realizadas después de 6 semanas de la ovariectomía o de la cirugía de simulacro (sham surgery), siendo los animales sacrificados después de 7, 14 y 28 días de la cirugía de implantación del biosilicato cristalino. Los resultados previos demostraron que el proceso de reparación alveolar de los animales utilizados en este estudio podría estar activo después de 6 semanas tras el trauma, lo que hace la elección de análisis hasta el final de la $4^{\mathrm{a}}$ semana, un período confiable para el análisis del comportamiento del biosilicato cristalino.

Schulte et al. (2013) evidenciaron a través de exámenes computarizados que, transcurridas 6 semanas de la cirugía de ovariectomía, las ratas presentaban una disminución acentuada en el trabeculado óseo y en la morfología ósea en la región femoral, validando el período de 6 semanas después de la cirugía de ovariectomía como un período adecuado y confiable para que los animales presenten un cuadro compatible con la osteoporosis.

La granulación ideal de los biomateriales particulados para su uso en el área odontológica es bastante discutida y varía de acuerdo con el biomaterial utilizado. Así, Fetner et al. (1994) evaluaron distintas granulaciones de 6 diferentes 
biovidrios, no encontrando diferencia en los resultados cuando los biomateriales evaluados medían entre 90 y 710 micrómetros. Hall et al. (1999) estudiaron el comportamiento de diferentes biomateriales en contacto con la superficie de implantes oseointegrados en la mandíbula de perros utilizando el biovidrio con granulación entre 300 y 355 micrómetros y observaron buen comportamiento de los biomateriales probados. Gu et al. (2013) estudiaron la implantación de diferentes biomateriales en la calota de ratas y encontraron los mejores resultados para el biovidrio con gránulos que presentaban un tamaño próximo a los 200 micrómetros. En nuestro estudio el tamaño de los gránulos osciló entre los 200 y los 300 micrómetros por la facilidad de manipulación del material y por ser posible capturar el biosilicato cristalino embebido en suero con el uso de un portaamalgamas infantil para insertarlo en el alvéolo, con una pérdida mínima de material y con facilidad para mantenerlo en la cavidad. No fue posible establecer la cantidad exacta insertada en cada alvéolo, siendo utilizado como parámetro el llenado total de la punta activa del instrumental. Este procedimiento fue considerado adecuado, pues durante los análisis histológicos, toda el área que se pretendía estudiar se encontraba rellenada con el biomaterial.

Los resultados del presente estudio pusieron de manifiesto un alto grado de biocompatibilidad y un excelente comportamiento del biosilicato cristalino. No se constató respuesta inflamatoria derivada de la implantación del biomaterial, estando nuestros resultados en concordancia con los de Granito et al. Estos autores evaluaron los efectos del Bioglass45S5® y del biosilicato en defectos óseos creados en tibia de ratas. La ausencia de reacción inflamatoria, así como de células gigantes, favorecieron la interacción hueso/implante. Este hecho concuerda con lo que se observó en otros trabajos realizados por el mismo grupo de investigación evaluando el comportamiento de diferentes biovidrios (da Rocha Barros et al., 2007; Azenha et al., 2010). Otras investigaciones también concluyeron que el biovidrio puede ser usado en diferentes situaciones clínicas con un comportamiento favorable y satisfactorio (Fernandes et al., 2017).

Estudios previos revelaron que la cristalización del biovidrio, disminuye el nivel de bioactividad, pudiendo incluso hacer un vidrio bioactivo en bioenergía (Li et al., 1992). Sin embargo, esto no se observó en el presente estudio. Contrariamente a estas afirmaciones, Hench \& West (1996) constataron que la cris- talización total del biovidrio promueve un aumento de su índice de bioactividad a un nivel superior a los de las vitrocerámicas comercializadas. Moura et al. demostraron en experimentos in vitro que el biosilicato $\left(\mathrm{P}_{2} \mathrm{O}_{5} \cdot \mathrm{Na}_{2} \mathrm{O} . \mathrm{CaO} \cdot \mathrm{SiO}_{2}\right)$ es altamente bioactivo, presentando mayor formación de matriz ósea mineralizada en sistema de cultivo de células osteogénicas en comparación con el biosilicato vítreo y al Bioglass $45 S 5^{\circledR}$. La cristalización total del biosilicato puede alterar importantes propiedades del material, modificando el $\mathrm{pH}$ del medio y haciéndolo alcalino, favoreciendo la diferenciación y la función osteoblástica (Xynos et al., 2000; Loty et al., 2001). La cristalización del biosilicato puede también alterar otras propiedades del material como el índice de disolución o su grado de solubilidad, interfiriendo en la formación ósea.

Vogel et al. (2004) indico que la formación de células gigantes multinucleadas evaluadas durante la degradación de partículas del Bioglass $45 S 5 \circledast$ en conejos era tiempo-dependiente y fue influenciada por la solubilidad del material implantado. En el presente estudio, cuando se evaluó el biosilicato cristalino implantado en el alvéolo de ratas ovariectomizadas no se observó degradación o disolución del material en los períodos analizados. Scotchford et al. (2011) analizaron la degradación de discos prefabricados que contenían biovidrio implantados en la calota de ratas, demostrando que, tras largos períodos experimentales, es posible encontrar áreas de los discos que sufrieron cambios y deformación, sugiriendo la sustitución del material implantado por tejido del lecho receptor en mayor intensidad a partir de la $8^{a}$ semana. Como en el presente trabajo, el período máximo de estudio fue de 28 días, no fue posible hacer comparaciones con los resultados encontrados por estos autores. Uno de los inconvenientes para realizar estudios con períodos de evaluación más largos es la dificultad de mantenimiento y cuidado de los animales.

Cuando se analizó el período de 7 días, los grupos 1 y 4 presentaron resultados superiores que los grupos 2 y 3 en cuanto a la formación ósea con diferencias estadísticamente significativas. En este período, las ratas cuyo alvéolo fue rellenado por coágulo fueron las que presentaron los resultados más favorables, lo que podría indicar que, en el período inicial de reparación alveolar, el biosilicato cristalino puede haber retrasado este proceso, independientemente de si la rata fue sometida a la cirugía de ovariectomía o cirugía de simulacro (sham surgery). 
Después del período de 14 días, las diferencias estadísticas se mantuvieron, con los grupos 1 y 4 y los grupos 2 y 3 entre sí. En este período es importante destacar que, incluso en los animales donde el biosilicato cristalino fue implantado, se encontró formación de hueso en, al menos el $25 \%$ del alvéolo; lo que ponía de manifiesto el buen comportamiento biológico del biosilicato. A los 28 días, las diferencias estadísticas permanecieron entre los mismos grupos estudiados. En los grupos implantados con el biosilicato, cerca del $60 \%$ del alvéolo ya había sido rellenado por hueso neoformado. Schulte et al. evaluaron el comportamiento de un biomaterial en el fémur de ratas osteoporóticas y concluyeron que las ratas osteoporóticas pueden presentar formación ósea similar a ratas sanas después de largos períodos (12 semanas). Los resultados satisfactorios del biosilicato cristalino después de 4 semanas apuntan a la tendencia de que, después de 12 semanas, la formación de hueso alcanzó resultados similares a los grupos cuyos alvéolos se rellenaron sólo por coágulo.

Durante todo el estudio, las partículas del biosilicato cristalino permanecieron en el alvéolo y fueron progresivamente siendo rodeadas por hueso neoformado, que también fue encontrado en íntimo contacto con la superficie del material. También se puede inferir que el biopuerto implantado ejerció actividad de estimulación ósea, donde es posible observar una recuperación acentuada en el volumen de hueso del $7^{\circ}$ para el $14^{\circ}$ día y del $14^{\circ}$ al $28^{\circ}$ día. Hubo una satisfactoria recuperación morfológica al final del estudio, incluso con la presencia del biomaterial en el alvéolo y con el mínimo de $60 \%$ del área estudiada siendo cubierta por hueso neoformado. Estos resultados son similares a los del reciente estudio de Biguetti et al. (2019). Matsumoto et al. (2012) encontraron una acentuada reparación ósea en el período de 14 días, únicamente en el grupo experimental del biovidrio, justificando la capacidad de osteoconducción y osteoinducción de este biomaterial. En nuestro estudio, el biosilicato cristalino ha ejercido solo la función de osteoconducción, no siendo posible afirmar si hubo inducción en la formación de tejido óseo. Los estudios anteriores demuestran que la intensidad de formación de nuevo hueso depende no solo de las características del material, sino también de la especie de animal utilizado, el sitio receptor, la cantidad y el tamaño de las partículas injertadas. La gran mayoría de los trabajos señalan resultados positivos de los biovidrios con una excelente capacidad osteoconductora, además de presentar un menor costo cuando es comparado con otros biovidrios y biomateriales.

\section{CONCLUSIONES}

Las conclusiones del presente estudio fueron:

$1^{\circ}$. Ningún animal presentó signos clínicos de inflamación o infección, necrosis u otras lesiones clínicas afectando a los tejidos blandos y/o óseos.

$2^{\circ}$. Los grupos de ratas con cirugía de simulacro + coágulo (grupo 1) y los de ratas con ovariectomía + biosilicato (grupo 4) fueron los que presentaron los mejores resultados histomorfométricos durante todo el estudio.

$3^{\circ}$. El biosilicato cristalino presentó un resultado favorable en la reparación ósea.

$4^{\circ}$. No se observó la reabsorción de las partículas del biosilicato cristalino tras 28 días de su implantación.

AZENHA, M. R.; MAGRO FILHO, O.; PEITL, O.; TORO, B. \& ARCHILLA A. R. Alveolar bone repair in ovarictomized rats with crystallized biosilicate histomorphometric analysis. Int. J. Odontostomat., 13(2):162-171, 2019.

ABSTRACT: The objective of this study was to assess the process of alveolar bone repair in rats subjected to sham surgery or ovariectomized rats, after alveolar filling with clot or with crystalline biosilicate. Sixty Wistar rats were divided into four groups $(n=15)$ according to the treatment: Group 1 - rats subjected to sham surgery with sockets filled with clot; Group 2- rats submitted to sham surgery with sockets filled with crystalline biosilicate; Group 3 ovariectomized rats with sockets filled with clot; Group 4 ovariectomized rats with sockets filled with crystalline biosilicate. After 7, 14 and 28 days, the animals were sacrificed, bone samples were taken, stained with hematoxylin-eosin and analyzed under a microscope to perform a histomorphometric analysis. The highest percentages of bone formation were presented in groups 1 (32\% at 7 days, $46 \%$ at 14 days and $83.5 \%$ at 28 days) and $4(27.1 \%$ at 7 days, $41.1 \%$ at 14 days and 79.7 $\%$ after 28 days). In the sockets filled with clot, the rats subjected to sham surgery showed the best results, while in the sockets filled with biosilicate, the ovariectomized rats had significantly higher percentages. In this study, the crystalline biosilicate behaved as an adequate biomaterial for bone repair, favoring osteoconduction.

KEY WORDS: bone remodeling, osteoporosis, rats Wistar, silicates.

\section{REFERENCIAS BIBLIOGRÁFICAS}

Azenha, M. R.; de Lacerda, S. A.; Marão, H. F.; Filho, O. P. \& Filho, O. M. Evaluation of crystallized biosilicate in the reconstruction of calvarial defects. J. Maxillofac. Oral Surg., 14(3):659-65, 2015. 
Azenha, M. R.; Peitl, O. \& Barros, V. M. Bone response to biosilicates with different crystal phases. Braz. Dent. J., 21(5):383-9, 2010.

Biguetti, C. C.; Cavalla, F.; Tim, C. R.; Saraiva, P. P.; Orcini, W.; De Andrade Holgado, L.; Rennó, A. C. M. \& Matsumoto, M. A. Bioactive glass-ceramic bone repair associated or not with autogenous bone: a study of organic bone matrix organization in a rabbit critical-sized calvarial model. Clin. Oral Investig., 23(1):413-21, 2019.

Costantino, P. D. \& Friedman, C. D. Synthetic bone graft substitutes. Otolaryngol. Clin. North Am., 27(5):1037-74, 1994.

Cypher, T. J. \& Grossman, J. P. Biological principles of bone graft healing. J. Foot Ankle Surg., 35(5):413-7, 1996

da Rocha Barros, V. M.; Liporaci, J. L. Jr.; Rosa, A. L.; Junqueira, M. C.; de Oliveira, P. T.; Johnson, A. \& van Noort, R. Bone response to three different chemical compositions of fluorcanasite glassceramic. J. Biomed. Mater. Res. A, 83(2):480-3, 2007.

Fernandes, K. R.; Magri, A. M. P.; Kido, H. W.; Parisi, J. R.; Assis, L.; Fernandes, K. P. S.; Mesquita-Ferrari, R. A.; Martins, V. C.; Plepis, A. M.; Zanotto, E. D.; et al. Biosilicate/PLGA osteogenic effects modulated by laser therapy: In vitro and in vivo studies. J. Photochem. Photobiol. B, 173:258-65, 2017.

Fetner, A. E.; Hartigan, M. S. \& Low, S. B. Periodontal repair using Perioglas in nonhuman primates: clinical and histologic observations. Compendium, 15(7):932, 935-8, 1994.

Granito, R. N.; Ribeiro, D. A.; Rennó, A. C.; Ravagnani, C.; Bossini, P. S.; Peitl-Filho, O.; Zanotto, E. D.; Parizotto, N. A. \& Oishi, J. Effects of biosilicate and bioglass $45 \mathrm{~S} 5$ on tibial bone consolidation on rats: a biomechanical and a histological study. J. Mater. Sci. Mater. Med., 20(12):2521-6, 2009.

Gu, Y.; Huang, W.; Rahaman, M. N. \& Day, D. E. Bone regeneration in rat calvarial defects implanted with fibrous scaffolds composed of a mixture of silicate and borate bioactive glasses. Acta Biomater., 9(11):9126-36, 2013.

Hall, E. E.; Meffert, R. M.; Hermann, J. S.; Mellonig, J. T. \& Cochran, D. L. Comparison of bioactive glass to demineralized freeze-dried bone allograft in the treatment of intrabony defects around implants in the canine mandible. J. Periodontol., 70(5):526-35, 1999.

Hasan, A.; Byambaa, B.; Morshed M.; Cheikh, M. I.; Shakoor, R. A.; Mustafy, T. \& Marei, H. E. Advances in osteobiologic materials for bone substitutes. J. Tissue Eng. Regen. Med., 12(6):144868, 2018.

Hench, L. L. \& West, J. K. Biological applications of bioactive glasses. Life Chem. Rep., 13:187-241, 1996.

Li, P.; Yang, Q.; Zhang, F. \& Kokubo, T. The effect of residual glassy phase in a bioactive glass-ceramic on the formation of its surface apatite layerin vitro. J. Mater. Sci. Mater. Med., 3(6):452-6, 1992.

Loty, C.; Sautier, J. M.; Tan, M. T.; Oboeuf M.; Jallot, E.; Boulekbache H.; Greenspan, D. \& Forest, N. Bioactive glass stimulates in vitro osteoblast differentiation and creates a favorable template for bone tissue formation. J. Bone Miner. Res., 16(2):231-9, 2001.

Martins, C. H.; Carvalho, T. C.; Souza, M. G.; Ravagnani, C.; Peitl, O.; Zanotto, E. D.; Panzeri, H. \& Casemiro, L. A. Assessment of antimicrobial effect of Biosilicate $\AA$ against anaerobic, microaerophilic and facultative anaerobic microorganisms. $J$. Mater. Sci. Mater. Med., 22(6):1439-46, 2011.

Matsumoto, M. A.; Caviquioli, G.; Biguetti, C. C.; Holgado, L. de A.; Saraiva, P. P.; Rennó, A. C. \& Kawakami, R. Y. A novel bioactive vitroceramic presents similar biological responses as autogenous bone grafts. J. Mater. Sci. Mater. Med., 23(6):1447-56, 2012.

Moura, J.; Teixeira, L. N.; Ravagnani, C.; Peitl, O.; Zanotto, E. D.; Beloti, M. M.; Panzeri, H.; Rosa, A. L. \& de Oliveira, P. T. In vitro osteogenesis on a highly bioactive glass-ceramic (Biosilicate). J. Biomed. Mater. Res. A, 82(3):545-57, 2007.

Pina, S.; Rebelo, R.; Correlo, V. M.; Oliveira, J. M. \& Reis, R. L. Bioceramics for osteochondral tissue engineering and regeneration. Adv. Exp. Med. Biol., 1058:53-75, 2018.
Roriz, V. M.; Rosa, A. L.; Peitl ,O.; Zanotto, E. D.; Panzeri, H. \& de Oliveira, P. T. Efficacy of a bioactive glass-ceramic (Biosilicate) in the maintenance of alveolar ridges and in osseointegration of titanium implants. Clin. Oral Implants Res., 21(2):148-55, 2010.

Schulte, F. A.; Ruffoni, D.; Lambers, F. M.; Christen, D.; Webster, D. J.; Kuhn, G. \& Müller, R. Local mechanical stimuli regulate bone formation and resorption in mice at the tissue level. PLoS One, 8(4):e62172, 2013.

Scotchford, C. A.; Shahtaheri, M.; Chen, P. S.; Evans, M.; Parsons, A. J.; Aitchison, G. A.; Efeoglu, C.; Burke, J. L.; Vikram, A.; Fisher, S. E.; et al. Repair of calvarial defects in rats by prefabricated, degradable, long fibre composite implants. J. Biomed. Mater. Res. A, 96(1):230-8, 2011.

Teófilo, J. M.; Azevedo, A. C.; Petenusci, S. O.; Mazaro, R. \& LamanoCarvalho, T. L. Comparison between two experimental protocols to promote osteoporosis in the maxilla and proximal tibia of female rats. Pesqui. Odontol. Bras., 17(4):302-6, 2003.

Tirapelli, C.; Panzeri, H.; Lara, E. H.; Soares, R. G.; Peitl, O. \& Zanotto, E. D. The effect of a novel crystallised bioactive glassceramic powder on dentine hypersensitivity: a long-term clinical study. J. Oral Rehabil., 38(4):253-62, 2011.

Vogel, M.; Voigt, C.; Knabe, C.; Radlanski, R. J.; Gross, U. M. \& Müller-Mai, C. M. Development of multinuclear giant cells during the degradation of Bioglass particles in rabbits. J. Biomed. Mater. Res. A, 70(3):370-9, 2004.

Wang, W. \& Yeung, K. W. K. Bone grafts and biomaterials substitutes for bone defect repair: A review. Bioact. Mater., 2(4):224-47, 2017.

Wu, T.; Cheng, N.; Xu, C.; Sun, W.; Yu, C. \& Shi, B. The effect of mesoporous bioglass on osteogenesis and adipogenesis of osteoporotic BMSCs. J. Biomed. Mater. Res. A, 104(12):300414, 2016.

Xynos, I. D.; Edgar, A. J.; Buttery, L. D.;Hench, L. L. \& Polak, J. M. Ionic products of bioactive glass dissolution increase proliferation of human osteoblasts and induce insulin-like growth factor II mRNA expression and protein synthesis. Biochem. Biophys. Res. Commun., 276(2):461-5, 2000.

Dirección para correspondencia:

Marcelo Rodrigues Azenha

Avenida do Café, s/n

Facultad de Odontología de Ribeirão Preto

Universidade de São Paulo

BRASIL

Email: marceloazenha@yahoo.com.br marceloazenha@usp.br

Recibido : 20-11-2018

Aceptado: 04-02-2019 